\title{
Renormalization group structure for sums of variables generated by incipiently chaotic maps
}

\author{
Miguel Angel Fuentes ${ }^{1,2,3}$ and Alberto Robledo ${ }^{4}$ \\ ${ }^{1}$ Santa Fe Institute, 1399 Hyde Park Road, Santa Fe, New Mexico 87501, USA \\ ${ }^{2}$ Centro Atómico Bariloche, Instituto Balseiro and CONICET, 8400 Bariloche, \\ Argentina \\ ${ }^{3}$ Center for Advanced Studies in Ecology and Biodiversity, Facultad de Ciencias \\ Biológicas, Pontificia Universidad Católica de Chile, Casilla 114-D, Santiago CP \\ 6513677, Chile \\ ${ }^{4}$ Instituto de Física, Universidad Nacional Autónoma de México, Apartado Postal \\ 20-364, México 01000 DF, Mexico \\ E-mail: fuentesm@santafe.edu
}

\begin{abstract}
We look at the limit distributions of sums of deterministic chaotic variables in unimodal maps and find a remarkable renormalization group (RG) structure associated to the operation of increment of summands and rescaling. In this structure - where the only relevant variable is the difference in control parameter from its value at the transition to chaos - the trivial fixed point is the Gaussian distribution and a novel nontrivial fixed point is a multifractal distribution that emulates the Feigenbaum attractor, and is universal in the sense of the latter. The crossover between the two fixed points is explained and the flow toward the trivial fixed point is seen to be comparable to the chaotic band merging sequence. We discuss the nature of the Central Limit Theorem for deterministic variables.
\end{abstract}




\section{Introduction}

As it is well documented [1, increasingly larger sums of iterates of chaotic mappings give rise to a Gaussian stationary distribution in the same way independent random variables do according to the ordinary central limit theorem [2, 3]. This deepseated property, remarkably shared by deterministic and random systems composed of essentially uncorrelated variables, naturally raises questions about the existence, and if so, uniqueness or diversity, of limit distributions for systems made up of deterministic correlated variables. Related to these issues recent [4]-[7] numerical explorations of time averages of iterates at the period-doubling transition to chaos [8, 9] have been presented. Since the trajectories linked to this critical attractor are nonergodic and nonmixing the question of whether there is such stationary distribution for sums of iterates at the transition to chaos holds added interest as it may provide new angles to appraise the statistical mechanical analogy that is found in chaotic dynamics [10].

The dynamics toward and at the Feigenbaum attractor is now known in much detail [10, 11], therefore, it appears feasible to analyze also the properties of sums of iterate positions for this classic nonlinear system with the same kind of analytic reasoning and numerical thoroughness. Here we present the results for sums of chronological positions of trajectories associated to quadratic unimodal maps. We consider the case of the sum of positions of trajectories inside the Feigenbaum attractor as well as those within the chaotic $2^{K}$-band attractors obtained when the control parameter is shifted to values larger than that at the transition to chaos. Time and ensemble averages differ at the transition to chaos and here we chose to study the time average of a single trajectory initiated inside the attractor since all such trajectories, as explained below, are simply related. Clearly, time and ensemble averages are equivalent for chaotic attractors. From the information obtained we draw conclusions on the properties of the stationary distributions for these sums of variables. Our results, that reveal a multifractal stationary distribution that mirrors the features of the Feigenbaum attractor, can be easily extended to other critical attractor universality classes and other routes to chaos. About the relevance of our findings to physical systems, it is interesting to note, as one example, the parallels that have been found to exist between the dynamics at the noise-perturbed onset of chaos in unimodal maps and the dynamics of glass formation [12]. In this connection chaotic band merging plays a central role in the relaxation properties of time correlation functions, while the multifractal attractor and multiband attractors in its neighborhood display the characteristic aging scaling property of glass formers [12].

The overall picture we obtain is effectively described within the framework of the renormalization group ( $\mathrm{RG}$ ) approach for systems with scale invariant states or attractors. Firstly, the RG transformation for the distribution of a sum of variables is naturally given by the change due to the increment of summands followed by a suitable restoring operation. Second, the limit distributions can be identified as fixed points reached according to whether the acting relevant variables are set to zero or not. Lastly, 
the universality class of the non-trivial fixed-point distribution can be assessed in terms of the existing set of irrelevant variables.

As it is well known [8, 9] a few decades ago the RG approach was successfully applied to the period-doubling route to chaos displayed by unimodal maps. In that case the RG transformation is functional composition and rescaling of the mapping and its effect re-enacts the growth of the period doubling cascade. In our case the RG transformation is the increment of terms and adjustment of the sum of positions and its effect is instead to go over again the merging of bands in the chaotic region.

Specifically, we consider the Feigenbaum map $g(x)$, obtained from the fixed point equation $g(x)=\alpha g(g(x / \alpha))$ with $g(0)=1$ and $g^{\prime}(0)=0$, and where $\alpha=-2.50290 \ldots$ is one of Feigenbaum's universal constants [8, 9]. (For expediency we shall from now on denote the absolute value $|\alpha|$ by $\alpha$ ). Numerically, the properties of $g(x)$ can be conveniently obtained from the logistic map $f_{\mu}(x)=1-\mu x^{2},-1 \leq x \leq 1$, with $\mu=$ $\mu_{\infty}=1.401155189092 \ldots$ The dynamics associated to the Feigenbaum map is determined by its multifractal attractor. For a recent detailed description of these properties see [10, 11]. For values of $\mu>\mu_{\infty}$ we employ a well-known scaling relation supported by numerical results.

Initially we present properties of the sum of the absolute values $\left|x_{t}\right|$ of positions $x_{t}=f_{\mu_{\infty}}\left(x_{t-1}\right), t=1,2,3, \ldots$, as a function of total time $N$ visited by the trajectory with initial position $x_{0}=0$, and obtain a patterned linear growth with $N$. We analyze this intricate fluctuating pattern, confined within a band of finite width, by eliminating the overall linear increment and find that the resulting stationary arrangement exhibits features inherited from the multifractal structure of the attractor. We derive an analytical expression for the sum that corroborates the numerical results and provide an understanding of its properties. Next, we consider the straight sum of $x_{t}$, where the signs taken by positions lessen the growth of its value as $N$ increases and the results are consistently similar to those for the sum of $\left|x_{t}\right|$, i.e. linear growth of a fixed-width band within which the sum displays a fluctuating arrangement. Numerical and analytical details for the sum of $x_{t}$ are presented. Then, we show numerical results for the sum of iterated positions obtained when the control parameter is shifted into the region of chaotic bands. In all of these cases the distributions evolve after a characteristic crossover towards a Gaussian form. Finally, we rationalize our findings in terms of an $\mathrm{RG}$ framework in which the action of the Central Limit Theorem plays a fundamental role and provide details of the crossover from multiband distributions to the gaussian distribution. We discuss our results. 


\section{Sums of positions at the chaos threshold}

\subsection{Sum of absolute values $\left|x_{t}\right|$}

The starting point of our study is the evaluation of

$$
y_{\mu}(N) \equiv \sum_{t=1}^{N}\left|x_{t}\right|,
$$

with $\mu=\mu_{\infty}$ and with $x_{0}=0$. Fig. $1 \mathrm{~A}$ shows the result, where it can be observed that the values recorded, besides a repeating fluctuating pattern within a narrow band, increase linearly on the whole. The measured slope of the linear growth is $c=0.56245 \ldots$ Fig. 1B shows an enlargement of the band, where some detail of the complex pattern of values of $y_{\mu_{\infty}}(N)$ is observed. A stationary view of the mentioned pattern is shown in Fig. 1C, where we plot

$$
y_{\mu_{\infty}}^{\prime}(N) \equiv \sum_{t=1}^{N}\left(\left|x_{t}\right|-c\right),
$$

in logarithmic scales. There, we observe that the values of $y_{\mu_{\infty}}^{\prime}(N)$ fall within horizontal bands interspersed by gaps, revealing a fractal or multifractal set layout. The top (zeroth) band contains $y_{\mu_{\infty}}^{\prime}$ for all the odd values of $N$, the 1st band next to the top band contains $y_{\mu_{\infty}}^{\prime}$ for the even values of $N$ of the form $N=2+4 m, m=0,1,2, \ldots$ The 2 nd band next to the top band contains $y_{\mu_{\infty}}^{\prime}(N)$ for $N=2^{2}+2^{3} m, m=0,1,2, \ldots$, and so on. In general, the $k$-th band next to the top band contains $y_{\mu_{\infty}}^{\prime}\left(2^{k}+2^{k+1} m\right), m=0,1,2, \ldots$ Another important feature in this figure is that the $y_{\mu_{\infty}}^{\prime}(N)$ for subsequences of $N$ each of the form $N=(2 l+1) 2^{k}, k=0,1,2, \ldots$, with $l$ fixed at a given value of $l=0,1,2, \ldots$, appear aligned with a uniform slope $s=-1.323 \ldots$ The parallel lines formed by these subsequences imply the power law $y_{\mu_{\infty}}^{\prime}(N) \sim N^{s}$ for $N$ belonging to such a subsequence.

It is known [10, 13] that these two characteristics of $y_{\mu_{\infty}}^{\prime}(N)$ are also present in the layout of the absolute value of the individual positions $\left|x_{t}\right|, t=1,2,3, \ldots$ of the trajectory initiated at $x_{0}=0$; and this layout corresponds to the multifractal geometric configuration of the points of the Feigenbaum's attractor, see Fig. 2. In this case, the horizontal bands of positions separated by equally-sized gaps are related to the set of period-doubling 'diameters' [8, 9] employed for the construction of the multifractal [11]. The identical slope shown in the logarithmic scales by all the position subsequences $\left|x_{t}\right|$, $t=(2 l+1) 2^{k}, k=0,1,2, \ldots$, each formed by a fixed value of $l=0,1,2, \ldots$, implies the power law $\left|x_{t}\right| \sim t^{s}, s=-\ln \alpha / \ln 2=-1.3236 \ldots$, as the $\left|x_{t}\right|$ can be expressed as $\left|x_{t}\right| \simeq\left|x_{2 l+1}\right| \alpha^{-k}, t=(2 l+1) 2^{k}, k=0,1,2, \ldots$, or, equivalently, $\left|x_{t}\right| \sim t^{s}$. Notice that the index $k$ also labels the order of the bands from top to bottom. The power law behavior involving the universal constant $\alpha$ of the subsequence positions reflect the approach of points in the attractor toward its most sparse region at $x=0$ from its most compact region, as the positions at odd times $\left|x_{2 l+1}\right|=x_{2 l+1}$, those in the top band, correspond to the densest region of the set.

Having uncovered the through manifestation of the multifractal structure of the

attractor into the sum $y_{\mu_{\infty}}^{\prime}(N)$ we proceed to derive this property and corroborate the 

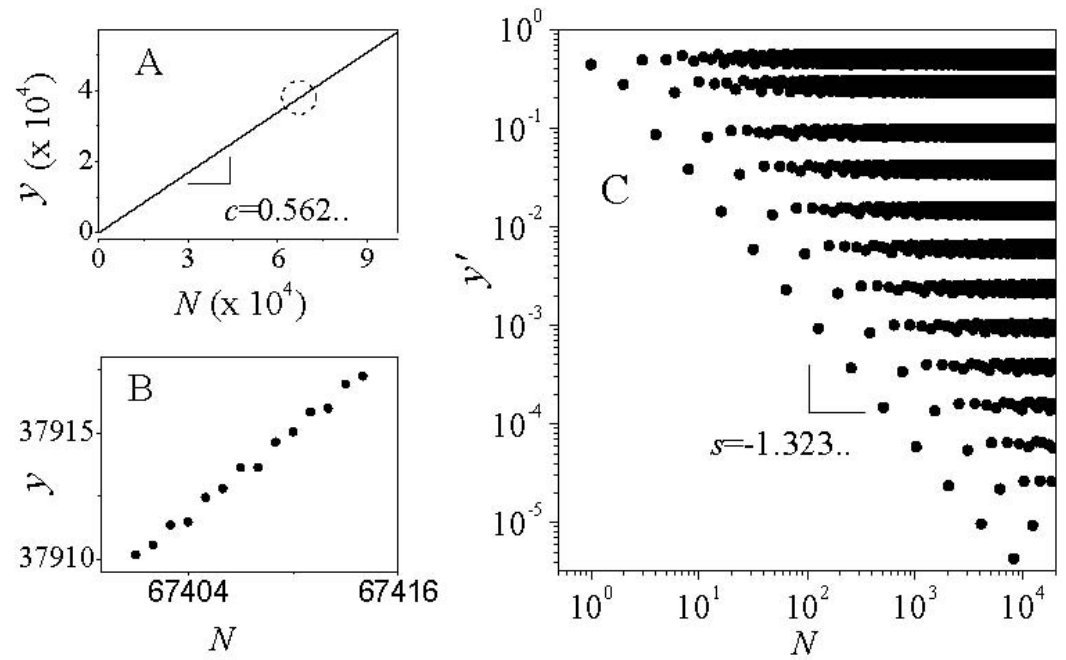

Figure 1. A) Sum $y_{\mu_{\infty}}(N)$ of absolute values of visited points $x_{t}, t=0, \ldots, N$, of the Feigenbaum's attractor with initial condition $x_{0}=0$. B) A closer look of the path of the sum (see dotted circle in A), for values of $N$ around 67410. C) Centered sum $y_{\mu_{\infty}}^{\prime}(N)$ in logarithmic scales. See text.

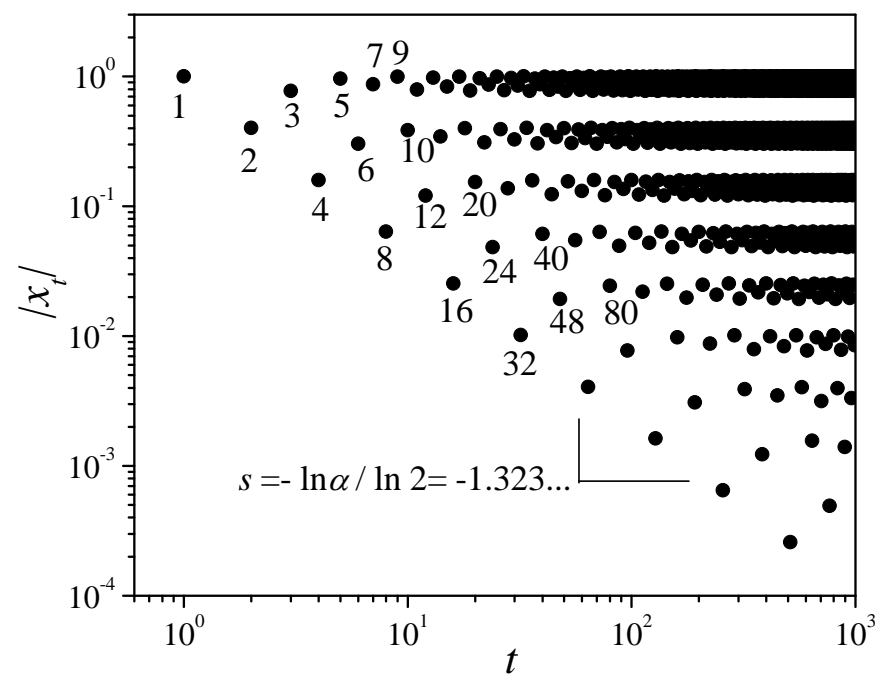

Figure 2. Absolute value of trajectory positions $x_{t}, t=0, \ldots$, for the logistic map $f_{\mu}(x)$ at $\mu_{\infty}$, with initial condition $x_{0}=0$, in logarithmic scale as a function of the logarithm of the time $t$, also shown by the numbers close to the points.

numerical evidence. Consider Eq. (1) with $N=2^{k}, k=0,1,2, \ldots$, the special case $l=0$ in the discussion above. Then the numbers of terms $\left|x_{t}\right|$ per band in $y_{\mu_{\infty}}\left(2^{k}\right)$ are: $2^{k-1}$ in the top band $(j=0), 2^{k-2}$ in the next band $(j=1), \ldots, 2^{0}$ in the $(k-1)$-th band, plus an additional position in the $k$-th band. If we introduce the average of the positions on 
the top band

$$
\langle a\rangle \equiv 2^{-(k-1)} \sum_{j=0}^{2^{k-1}} x_{2 j+1},
$$

the sum $y_{\mu_{\infty}}\left(2^{k}\right)$ can be written as

$$
y_{\mu_{\infty}}\left(2^{k}\right)=\langle a\rangle 2^{k-1} \sum_{j=0}^{k-2}(2 \alpha)^{-j}+\alpha^{-(k-1)}+\alpha^{-k} .
$$

Doing the geometric sum above and expressing the result as $y_{\mu_{\infty}}\left(2^{k}\right)=c 2^{k}+d \alpha^{-k}$, we have

$$
c=\frac{\langle a\rangle \alpha}{2 \alpha-1}, \quad d=\left(1-\frac{\langle a\rangle 2 \alpha}{2 \alpha-1}\right) \alpha+1 .
$$

Evaluation of Eq. (3) yields to $\langle a\rangle=0.8999 \ldots$, and from this we obtain $c=0.56227 \ldots$ and $d=0.68826 \ldots$ We therefore find that the value of the slope $c$ in Fig. $1 \mathrm{~A}$ is properly reproduced by our calculation. Also, since $\ln \left[y_{\mu_{\infty}}\left(2^{k}\right)-c 2^{k}\right]=\ln d-k \ln \alpha$, or, equivalently, $\ln y_{\mu_{\infty}}^{\prime}(N)=\ln d-N \ln \alpha / \ln 2, N=2^{k}, k=0,1,2, \ldots$, we corroborate that the value of the slope $s$ in the inset of Fig. $1 \mathrm{C}$ is indeed given by $s=-\ln \alpha / \ln 2=$ $1.3236 \ldots$ (We have made use of the identity $\alpha^{-k}=N^{-\ln \alpha / \ln 2}, N=2^{k}, k=0,1,2, \ldots$ ).

\subsection{Sum of values of $x_{t}$}

When considering the signs taken by positions $x_{t}$ we note that their sum,

$$
z_{\mu}(N) \equiv \sum_{t=0}^{N} x_{t}
$$

when $N=2^{k}, \mu=\mu_{\infty}$ and $x_{0}=0$, can be immediately obtained from the above derivation for $y_{\mu_{\infty}}\left(2^{k}\right)$ simply by replacing $\alpha^{-j}$ by $(-1)^{j} \alpha^{-j}$, as the $x_{t}$ of different signs of the trajectory starting at $x_{0}=0$ fall into separate alternating bands (described above and shown in Fig. 2). In short, $x_{t} \simeq(-1)^{j} x_{2 l+1} \alpha^{-j}, t=(2 l+1) 2^{k}, k=0,1,2, \ldots$ Writing $z_{\mu_{\infty}}\left(2^{k}\right)$ as $z_{\mu_{\infty}}\left(2^{k}\right)=e 2^{k}+f(-1)^{k} \alpha^{-k}$, we have

$$
e=\frac{\langle a\rangle \alpha}{2 \alpha+1}, \quad f=\left(1-\frac{\langle a\rangle 2 \alpha}{2 \alpha+1}\right)(-\alpha)+1 .
$$

Use of $\langle a\rangle=0.8999 \ldots$, leads to $e=0.37503 \ldots$ and $f=0.37443 \ldots$ Since $\ln \left(z_{\mu_{\infty}}\left(2^{k}\right)-e 2^{k}\right)=\ln f-k \ln \alpha$, or $\ln z_{\mu_{\infty}}^{\prime}(N)=\ln f-N \ln \alpha / \ln 2, N=2^{k}, k=$ $0,1,2, \ldots$, where $z_{\mu_{\infty}}^{\prime}\left(2^{k}\right) \equiv z_{\mu_{\infty}}\left(2^{k}\right)-e 2^{k}$, we obtain for the value of the slope $s^{\prime}$ associated to the plot of $z_{\mu_{\infty}}^{\prime}(N) \sim N^{s^{\prime}}$ in logarithmic scales the number $s^{\prime}=$ $-\ln \left(\alpha^{2}-1\right) \ln \alpha / \ln 2=-2.1984 \ldots$ (where the factor $\ln \left(\alpha^{2}-1\right)$ takes into account the fact that consecutive values of the same sign in $z_{\mu_{\infty}}^{\prime}\left(2^{k}\right)$ have $k=2 m$ or $2 m+1$, $m=0,1,2, \ldots)$ Our numerical evaluations for $z_{\mu_{\infty}}(N)$ and $z_{\mu_{\infty}}^{\prime}(N)$, shown in Fig. 3, reproduce the values given above for the slopes $e$ and $s^{\prime}$. Therefore, our numerical and analytical results are in agreement also in this case.

The relationship between the sum $y_{\mu_{\infty}}(N)$ with initial condition $x_{0}=0$ and all other sums $Y_{\mu_{\infty}}(M)$ of consecutive positions with any initial condition $x_{0}$ inside the attractor 

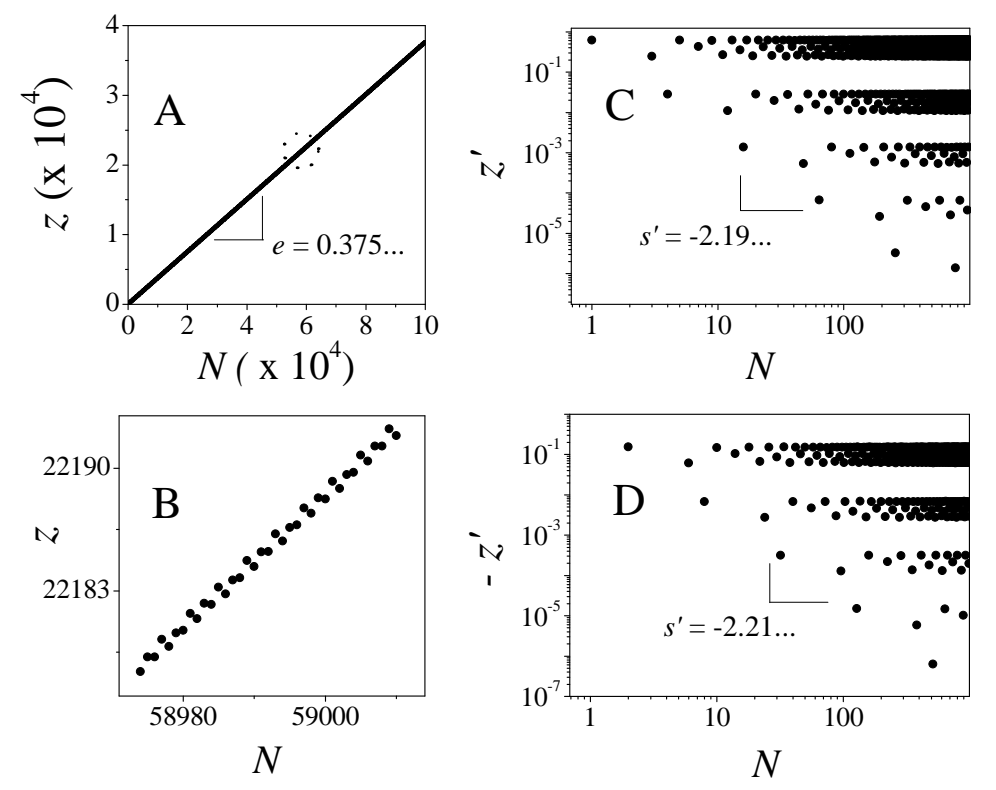

Figure 3. A) Sum $z_{\mu_{\infty}}(N)$ of values of visited points $x_{t}, t=0, \ldots, N$, of the Feigenbaum's attractor with initial condition $x_{0}=0$. B) A closer look of the path of the sum (see dotted circle in A), for values of $N$ around 59000. C and D) Centered sum $z_{\mu_{\infty}}^{\prime}(N)$ in logarithmic scales. See text.

can be obtained by inspection of Fig. 2. The sum $Y_{\mu_{\infty}}(M)$ differs only from $y_{\mu_{\infty}}(N)$ in the initial and final consecutive terms, $\sum_{t=1}^{t=t_{0}}\left|x_{t}\right|$ and $\sum_{t=N}^{t=M}\left|x_{t}\right|$, respectively, where $t_{0}$ is the time (shown in Fig. 2) at which the position $x_{0}$ is visited by the trajectory initiated at the origin $x=0$. When $t_{0}, N$, and $M$ are all powers of 2 the differences between the sums become simpler and expressable in terms of $y_{\mu_{\infty}}(N)$. When $N \rightarrow \infty$ and $M \rightarrow \infty$ the difference between them is only a finite term $y_{\mu_{\infty}}\left(t_{0}\right)$. Similar properties hold for the equivalent sums $z_{\mu_{\infty}}(N)$ and $Z_{\mu_{\infty}}(M)$ that take into account the signs of positions $x_{t}$.

\section{Sums of positions for chaotic bands}

We turn now to study the sum of positions of trajectories when $\Delta \mu \equiv \mu-\mu_{\infty}>0$. We recall that in this case the attractors are made up of $2^{K}, K=1,2,3, \ldots$, bands and that their trajectories consist of an interband periodic motion of period $2^{K}$ and an intraband chaotic motion. We evaluated numerically the sums $z_{\mu}(N)$ for a single trajectory with initial condition $x_{0}=0$ for different values of $\Delta \mu$. The sum $z_{\mu}^{\prime}(N)$ was then obtained similarly to Eq. (22) by substracting the average $\left\langle z_{\mu}(N)\right\rangle_{x_{0}}$ and rescaling with a factor $N^{-1 / 2}$. The panels in Fig. 4 show the evolution of the distributions for increasing number of summands $N$ for a value of $\Delta \mu$ (chosen for visual clarity) when the 


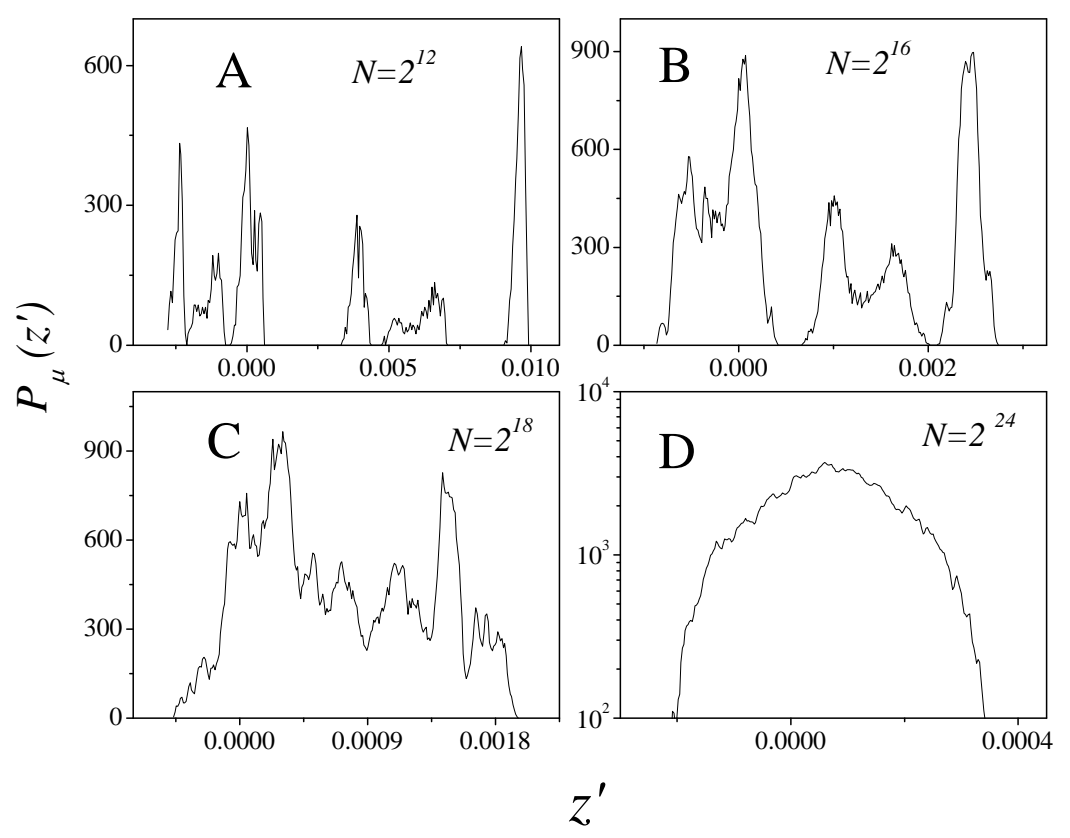

Figure 4. Distributions for the sums of positions $x_{t}, t=0, \ldots, N$, of a single trajectory with initial condition $x_{0}=0$ within the $2^{3}$-band attractor at $\Delta \mu=0.0028448109$. The number of summands $N$ are indicated in each panel. See text.

attractor consists of $2^{3}$ chaotic bands. Initially the distributions are multimodal with disconnected domains, but as $N$ increases we observe merging of bands and development of a single-domain bell-shaped distribution that as $N \longrightarrow \infty$ converges in all cases to a Gaussian distribution. As a check of the ergodic property of chaotic band attractors we have also evaluated the distributions of sums of positions starting with an ensemble of uniformly-distributed initial positions $x_{0}$ and obtained results equivalent in all respects to those shown in Fig. 4. Faster convergence to the Gaussian distribution is achieved in this latter case.

These numerical results can be understood as follows. We recollect [8, 9] that the relationship between the number of bands, $2^{K}, K \gg 1$, of a chaotic attractor and the control parameter distance $\Delta \mu$ at which it is located is given by $2^{K} \sim \Delta \mu^{-\kappa}$, $\kappa=\ln 2 / \ln \delta_{F}$, where $\delta_{F}=0.46692 \ldots$ is the universal constant that measures both the rate of convergence of the values of $\mu$ at period doublings or at band splittings to $\mu_{\infty}$. For $\Delta \mu$ small and fixed, the sum of sequential positions of the trajectory initiated at $x_{0}=0$, Eq. (1), exhibits two different growth regimes as the total time $N$ increases. To specify them we introduce the difference in value $\delta x_{t} \equiv x_{t}(\mu)-\bar{x}_{t}(\mu)$ between the position at time $t$ and the average position within the band $\bar{x}_{t}(\mu)$ occupied at time t. Clearly, when $K \gg 1$ the average positions $\bar{x}_{t}(\mu)$ approximate the multifractal positions $x_{t}\left(\mu_{\infty}\right)$ for $t \leq 2^{K}$. In the first regime, when $N \ll 2^{K}$, the properties of 
the sum $z_{\mu}(N)$ do not differ qualitatively from those of $z_{\mu_{\infty}}(N)$. This is because the fine structure of the Feigenbaum attractor is not suppressed by the fluctuations $\delta x_{t}$, as these contribute to the sum individually during the first cycle of the interband periodic motion. The discrete multi-scale nature of the distribution for $\mu_{\infty}$ is preserved when the interband motion governs the sum $z_{\mu}(N)$. The distributions for $z_{\mu}^{\prime}(N)$ and $z_{\mu_{\infty}}^{\prime}(N)$ are indistinguishable. In the second regime, when $N \gg 2^{K}$, the situation is opposite, after many interband cycles the fluctuations $\delta x_{t}$ add up in the sum and progressively wipe up the fine structure of the Feigenbaum attractor, leading to merging of bands and to the dominance of the fluctuating intraband motion. Ultimately, as $N \longrightarrow \infty$ the evolution of the distribution is similar to the action of the Central Limit Theorem and leads to a Gaussian stationary end result. It is also evident that as $\Delta \mu$ increases the first regime is shortened at the expense of the second, whereas when $\Delta \mu \longrightarrow 0$ the converse is the case. Therefore there exists an unambiguous $\Delta \mu$-dependent crossover behavior between the two radically different types of stationary distributions. This crossover is set out when the $\delta x_{t}$ fluctuations begin removing the band structure in $z_{\mu}^{\prime}(N)$ and ends when

these fluctuations have broadened and merged all the chaotic bands and $z_{\mu}^{\prime}(N)$ forms a single continuous interval. When $\mu=\mu_{\infty}$ this process never takes place.

\section{An RG approach for sums of positions}

We are in a position now to put together the numerical and analytical information presented above into the general framework of the RG approach. As known, this method was designed to characterize families of systems containing amongst their many individual states (or in this case attractors) a few exceptional ones with scale invariant properties and common to all systems in the family. We recall [14] that in the language of a minimal RG scheme there are two fixed points, each of which can be reached by the repeated application of a suitable transformation of the system's main defining property. One of the fixed points, is termed trivial and is reached via the RG transformation for almost all initial settings. i.e. for all systems in the family when at least one of a small set of variables, named relevant variables, is nonzero. To reach the other fixed point, termed nontrivial, it is necessary that the relevant variables are all set to zero, and this implies a severely restricted set of initial settings that ensure such critical RG paths. The nontrivial fixed point embodies the scale invariant properties of the exceptional state that occurs in each system in the family and defines a universality class, while the differences amongst the individual systems are distinguished through a large set of so-called irrelevant variables. The variables in the latter set gradually vanish as the RG transformation is applied to a system that evolves toward the nontrivial fixed point. Further, when any system in the family is given a nonzero but sufficiently small value to (one or more of) the relevant variables, the RG transformation converts behavior similar to that of the nontrivial fixed point into that resembling the trivial fixed point through a well-defined crossover phenomenon. 


\subsection{RG transformation and fixed points}

The recognition of the $\mathrm{RG}$ framework in the properties of the sums of positions of trajectories in unimodal maps and their associated distributions is straightforward. It can be concluded right away that in this problem (as defined here) there is only one relevant variable, the control parameter difference $\Delta \mu$. There is an infinite number of irrelevant variables, those that specify the differences between all possible unimodal maps (with quadratic maximum) and the Feigenbaum map $g(x)$. The RG transformation consists of the increment of one or more summands in the sum (1) followed by centering like in Eq. (2). The effect of the transformation in the distribution of the sum is then recorded. For sums of independent variables the transformation is equivalent to the convolution of distributions [15]. Notice that the transformation involves no scaling for the sums of positions at $\mu_{\infty}$. Examination of either Fig. 1 or Fig. 3 indicates that the values of the sums $y_{\mu_{\infty}}^{\prime}(N)$ and $z_{\mu_{\infty}}^{\prime}(N)$ are contained within a band of fixed width for all $N$ and therefore there should not be any scaling for such sums. The Feigenbaum attractor is not chaotic and its positions $x_{t}$ are not random variables. On the contrary the values of the sums $y_{\mu}^{\prime}(N)$ and $z_{\mu_{\infty}}^{\prime}(N)$ with $\mu>\mu_{\infty}$ spread as $N$ increases and scaling with a factor $N^{-1 / 2}$ maintains their values contained for $N \gg 1$. In this case the $x_{t}$ behave as independent random variables. There are two fixed-point distributions, the trivial continuum-space Gaussian distribution and the nontrivial discrete-space multifractal distribution (as observed in Figs. 1C, 3C and 3D). As explained above, there is a distinct crossover link between the two fixed-point distributions. Our results correspond to the dynamics inside the attractors, however, if the interest lies in considering only the stationary distribution of sums that do not contain the transient behavior of trajectories in their way to the attractor [4]-[6] our results are expected to give the correct answers for this case (see the Summary and discussion).

\subsection{Crossover via band merging}

In Fig. 5 we show a sector of the chaotic bands for the logistic map $f_{\mu}(x)$ where we indicate the widths of these bands at the control parameter values $\widehat{\mu}_{K}$ when they split each into two new bands. Interestingly, if we assume that for a given value of $K$ the widths of comparable lengths have equal lengths then these widths can be obtained from the widths of shortest and longest lengths via a simple scale factor consisting of an inverse power of $\alpha$. See Fig. 5. This introduces some degeneracy in the widths that propagates across the band splitting structure. Specifically, the widths scale now with increasing $K$ according to a binomial combination of the scaling of those that converge to the most crowded and most sparse regions of the multifractal attractor at $\mu_{\infty}$. As seen in Fig. 5 the widths form a Pascal triangle across the band splitting cascade. The total length $L_{K}$ of such chaotic $2^{K}$-band attractor can be immediately evaluated to yield

$$
L_{K}=\left(\alpha^{-1}+\alpha^{-2}\right)^{K} \text {. }
$$




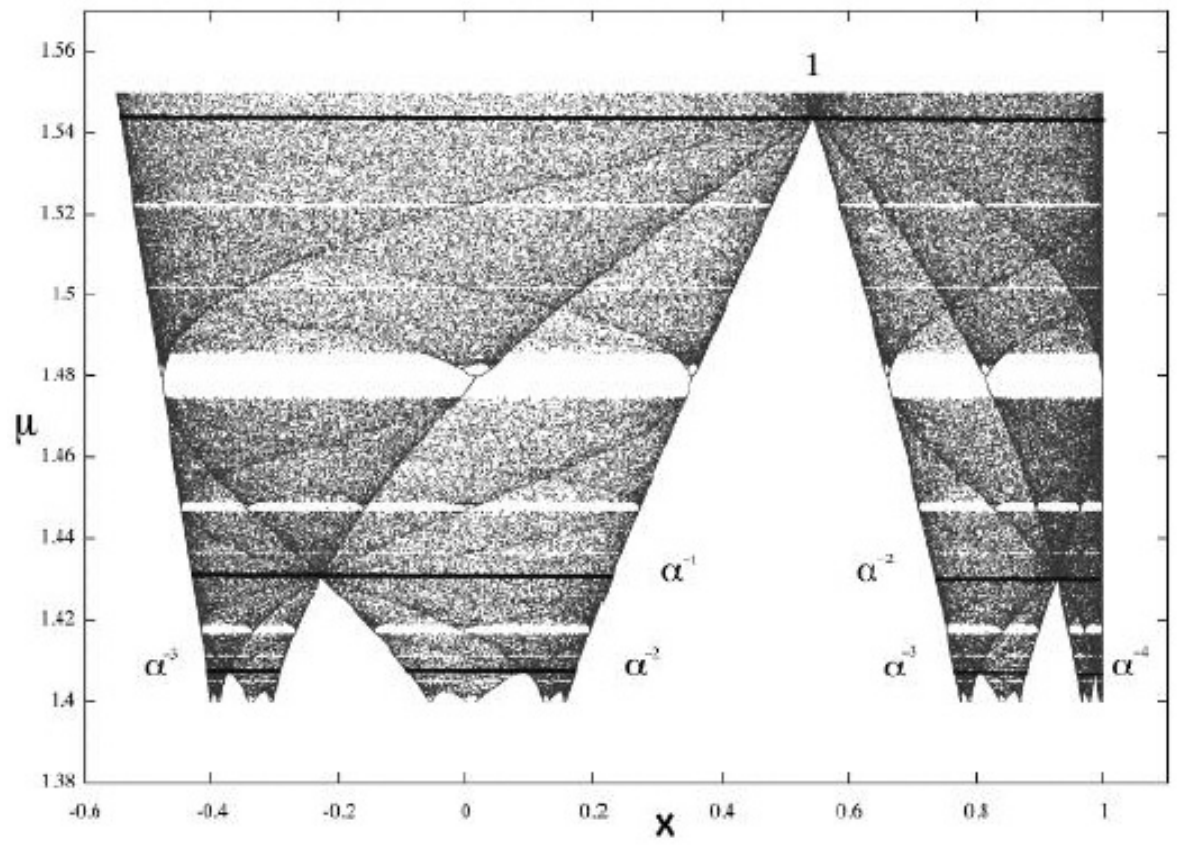

Figure 5. Sector of the band splitting cascade for the logistic map $f_{\mu}(x)$ that shows the formation of a Pascal triangle of band widths (blue lines) at splitting according to the scaling approximation explained in the text, where $\alpha \simeq 2.5091$ is the absolute value of Feigenbaum's universal constant.

(See Ref. [11] for a similar evaluation relating to the supercycle diameters occurring across the bifurcation tree for $\mu<\mu_{\infty}$ ).

Now, the sum $z_{\mu}(N)$ can be split into two terms, $z_{\mu}(N)=\bar{z}_{\mu}(N)+\delta z_{\mu}(N)$,

$$
\bar{z}_{\mu}(N)=\sum_{t=0}^{N} \bar{x}_{t} \quad, \quad \delta z_{\mu}(N)=\sum_{t=0}^{N} \delta x_{t} .
$$

Similarly to what we have seen for the sums $y_{\mu_{\infty}}(N)$ and $z_{\mu_{\infty}}(N)$, the first term $\bar{z}_{\mu}(N)$ is made of a narrow band of fixed width that shifts altogether linearly with $N$ to larger values while the band consists of a pattern of period $2^{K}$. Clearly $\bar{z}_{\mu}(N)$ does not participate in the band merging process, it is the second term $\delta z_{\mu}(N)$ that fluctuates and accomplishes band merging. Considering that all the correlated motion of $x_{t}$ has been taken over by $\bar{z}_{\mu}(N)$ the fluctuations of $\delta z_{\mu}(N)$ correspond to those of independent variables and band enlargement is measured by the mean square root displacement

$$
\left\langle\left[\delta z_{\mu}(N)\right]^{2}\right\rangle^{1 / 2} \sim N^{1 / 2}
$$

We can estimate the number of summands $N_{K}$ necessary to achieve the merging of $2^{K}$ bands into a single one by matching the two lengths $\left\langle\left[\delta z_{\mu}\left(N_{K}\right)\right]^{2}\right\rangle^{1 / 2}$ and $1-L_{K}$. From Eqs. (8) and (10) we obtain

$$
N_{K} \sim\left(\alpha^{-1}+\alpha^{-2}\right)^{K}\left[1-\left(\alpha^{-1}+\alpha^{-2}\right)\right]
$$


and considering $N_{K}$ to be of the form $N_{K}=2^{n_{K}}$ with $K \gg 1$ we obtain

$$
n_{K} \sim 2 K \ln \left[\left(\alpha^{-1}+\alpha^{-2}\right) / 2\right] .
$$

The crossover estimate in Eq. (12) for multiband into single-band distributions coincides in order of magnitude with the number of summands found necessary in Refs. [5] and [6] for numerical observations of long-tailed distributions resembling the so-called $q$ Gaussians [5].

\section{Summary and discussion}

In summary, we have found that the stationary distribution of the sum of iterate positions within the Feigenbaum attractor has a multifractal structure stamped by that of the initial multifractal set, while that involving sums of positions within the attractors composed of $2^{K}$ chaotic bands is the Gaussian distribution. We considered only the properties of a single sequence. At this transition the dynamics is nonergodic and nonmixing, therefore the two natural options, (i) time average with fixed initial condition and (ii) ensemble average of initial conditions at a large fixed time, are nonequivalent. A third option is to perform both ensemble and time averages. We chose option (i) because all sums with fixed initial positions within the attractor are simply related to each other via a deterministic term (as explained in Section II). Also, option (i) allowed us to obtain analytical results in closed form. For the ergodic chaotic band attractors the choice of a single initial condition leads to the same result as the use of an ensemble.

In Refs. [4]-6] sums of subsequent values of trajectories with uniformly distributed initial values across $-1 \leq x \leq 1$ were computed, and their properties were studied after discarding long transients. In Ref. [6] transients were discarded of lengths ranging from 2048 to 65536 and their results are reported to be insensitive to the transient length. A cursory inspection of Ref. [1] (with regards to Figs. 14 to 18 and the text associated to them) makes it evident that after iteration times smaller than the smaller transient discarded in Ref. [6] the trajectories considered have for all purposes fallen into the attractor. Thus, these sums are exceptionally well reproduced by sums of subsequent values $x_{t}$ with initial values inside the attractor. Next, when it is taken into account that sums belonging to every such initial value are simply related to that with $x_{0}=0$, it follows that the choice of sums we considered here capture the limiting properties of the sums studied in [4]-[6]. The numerical results that approximate $q$-Gaussians and/or Lévy distributions in [4]-7] may be understood by observing that the power laws in the $\Delta \mu=0$ multifractal distribution (see Fig. 3) are preserved at the tails of the distributions throughout the band-merging crossover. Only in the limit $N \rightarrow \infty$ the distribution tails become truly exponential.

We have also shown that the entire problem can be couched in the language of the RG formalism [15] in a way that makes clear the identification of the existing stationary distributions and the manner in which they are reached. These basic features suggest a 
degree of universality, and therefore limited to the critical attractor under consideration, in the properties of sums of deterministic variables at the transitions to chaos. Namely, the sums of positions of memory-retaining trajectories evolving under a vanishing Lyapunov exponent appear to preserve the particular features of the multifractal critical attractor under examination. Thus we expect that varying the degree of nonlinearity of a unimodal map would affect the scaling properties of time averages of trajectory positions at the period doubling transition to chaos, or alternatively, that the consideration of a different route to chaos, such as the quasiperiodic route, would lead to different scaling properties of comparable time averages. For instance, the known dependence of the universal constant $\alpha$ on the degree of nonlinearity $\varsigma$ of a unimodal map would show as a $\varsigma$-dependent exponents $s$ and $s^{\prime}$ that control the scale invariant property of the sums of trajectory positions with $x_{0}=0$ (shown in Figs. 1C, 3C and 3D).

It is worthwhile expanding here our previous comment about the applicability of our method to other maps. For instance, the stationary distributions associated to the prototypical circle map [8, 9] could be determined similarly, thus extending our study to the route to chaos via quasi-periodicity. The dynamics at the classic golden mean critical attractor of the circle map exhibits counterparts with the Feigenbaum attractor concerning all the basic features that we have made use of here [16]. This can be corroborated via comparison of Fig. 2 with Fig. 3 of [16] and related text therein.

We have contributed to clarify the nature and the circumstances under which a stationary distribution with universal properties (in the RG sense) may arise from sums of deterministic variables at the transition between regular and chaotic behavior, such as those studied here for variables evolving at zero Lyapunov exponent. In the absence of the fluctuating element present in chaotic attractors the distribution of the sums of these variables remains defined on a discrete multifractal set and is kept away from becoming a known (Gaussian or otherwise) continuum-space limit distribution for random variables.

Acknowledgements. We appreciate partial financial support by DGAPA-UNAM and CONACYT (Mexican agencies). AR is grateful for hospitality received at the SFI.

[1] Mackey M C and Tyran-Kaminska M 2006 Phys. Rep. 422167.

[2] van Kampen N G 1981 Stochastic Processes in Physics and Chemistry (North-Holland, Amsterdam).

[3] Khinchin A Y 1949 Mathematical Foundations of Statistical Mechanics (Dover, New York).

[4] Tirnakli U, Beck C and Tsallis C 2007 Phys. Rev. E 75 040106(R).

[5] Tirnakli U, Tsallis C and Beck C 2009 Phys. Rev. E 79056209.

[6] Tirnakli U, Tsallis C and Beck C (arXiv:0906.1262) [cond-mat.stat-mech].

[7] Grassberger P 2009 Phys. Rev. E 79057201.

[8] Schuster H G 1988 Deterministic Chaos. An Introduction (2nd Revised Edition, VCH Publishers, Weinheim).

[9] Hu B 1982 Phys. Rep. 91233.

[10] Mayoral E and Robledo A 2005 Phys. Rev. E 72026209.

[11] Robledo A and Moyano L G 2008 Phys. Rev. E 77036213 1-14. 
[12] Baldovin F and Robledo A 2005 Phys. Rev. E 72066213.

[13] Robledo A 2006 Physica A $\mathbf{3 7 0} 449$.

[14] Fisher M E 1998 Rev. Mod. Phys. 70653.

[15] A helpful note about the differences between the original RG transformation for unimodal maps [8, 9] and that presented here may be useful for the casual reader. (i) Feigenbaum's RG transformation consists of functional composition (and fixed scaling with the universal constant $\alpha$ ), while ours is summation of variables (with no scaling at the transition and scaling with the inverse square root of total number of terms beyond the transition). (ii) The fixed points in Feigenbaum's RG are maps, while the fixed points in ours are distributions. (iii) Feigenbaum's RG generates the period doubling cascade, while ours produces band merging. (iv) The purpose of Feigenbaum's RG is to characterize the critical attractor, while ours is to characterize stationary distributions.

[16] Hernández-Saldaña H and Robledo A 2006 Physica A 370286. 